\title{
Molecular Dynamics Study on Mechanical Properties in the Structure of Self-Assembled Quantum Dot
}

\author{
Tatsuya Yamaguchi ${ }^{1}$, Ken-ichi Saitoh ${ }^{2 *}$ \\ ${ }^{1}$ System Design Major, Graduate School of Science and Engineering, Kansai University, Suita, Japan \\ ${ }^{2}$ Department of Mechanical Engineering, Faculty of Engineering Science, Kansai University, Suita, Japan \\ Email: ${ }^{*}$ saitou@kansai-u.ac.jp
}

Received November 7, 2012; revised December 1, 2012; accepted December 8, 2012

\begin{abstract}
Stress and strain in the structure of self-assembled quantum dots constructed in the $\mathrm{Ge} / \mathrm{Si}(001)$ system is calculated by using molecular dynamics simulation. Pyramidal hut cluster composed of Ge crystal with $\{105\}$ facets surfaces observed in the early growth stage are computationally modeled. We calculate atomic stress and strain in relaxed pyramidal structure. Atomic stress for triplet of atoms is approximately defined as an average value of pairwise (virial) quantity inside triplet, which is the product of vectors between each two atoms. Atomic strain by means of atomic strain measure (ASM) which is formulated on the Green's definition of continuum strain. We find the stress (strain) relaxation in pyramidal structure and stress (strain) concentration in the edge of pyramidal structure. We discuss size dependency of stress and strain distribution in pyramidal structure. The relationship between hydrostatic stress and atomic volumetric strain is basically linear for all models, but for the surface of pyramidal structure and Ge-Si interface. This means that there is a reasonable correlation between atomic stress proposed in the present study and atomic strain measure, ASM.
\end{abstract}

Keywords: Molecular Dynamics; Self-Assembled Quantum Dot; Germanium; Silicon; Mechanical Property

\section{Introduction}

The quantum dot (QD) is a nanostructure which is assembled from a few thousand to a few ten of thousand semiconductor atoms such as silicon $(\mathrm{Si})$ and germanium (Ge). This micro-scale structure sometimes shows quantum size effect such that carriers or electrons are confined in very small dimensions. Therefore, previous studies have been developed so as to be applied to highperforming electronic devices, the quantum dot laser, the quantum dot solar cell, and so on.

In order to effectively utilize the special performance of quantum dots (QDs) for actual device behavior, we need to obtain high-performance QDs by reducing their size to approximately $15-20 \mathrm{~nm}$ (comparable with deBroglie wave length) or less in $x, y$ and $z$ directions. In addition, it is also important that, to some extent, QDs do not include any crystal defect or any impurity atom which would disturb the uniformity of the laser wave. For the purpose of satisfying these requirements in the industrial production, there is a self-organized growth method. Formation of three-dimensional island, called Stranski-Krastanov (S-K) growth, is caused by strain during epitaxial growth due to lattice mismatch between

"Corresponding author. different crystals in the substrate and the epitaxial layers. This method has the advantage that it is quite easy to form dense array of QDs. However, the shortage of uniformity in the cluster arrays, often called array defect, and the dispersion of cluster sizes are still today's issues [1]. Moreover, there is the lack of clarity in understanding detailed mechanical properties of QDs. Unique residual stress and strain distribute in QDs created by S-K growth. Indeed, atomic configuration and strain field in QDs is important because they affect QD's electronic structure and mechanical property, so the analysis of $\mathrm{X}$-ray diffraction and electron scattering experiment have been conducted. As a result, some understanding in regard with the appearance of dot positions and their configurational correlation has been done and creation of an equilibrium dot shape becomes possible. From now on, QD will widely prevail as a new nano material and device architecture, so we should try to evaluate QD's mechanical properties.

There have been studies on measurement of strain in QDs by using RHEED or XRD [2,3], but these provide only the average and qualitative estimation. In the case when experimental measurement is difficult, the computer simulation is often helpful and gives certain insight. There have been the studies on calculating the dis- 
tribution of strain and stress in $\mathrm{Ge}$ islands on the $\mathrm{Si}(001)$ surface by means of atomistic simulation [4-6]. Also, there have been the studies on $\mathrm{Ge} / \mathrm{Si}(001)$ system as the model system of hetero-epitaxial growth. Mo et al. successfully captured the STM images of Ge surfaces growing three-dimensionally on $\mathrm{Si}(001)$ substrate [7]. In their work, they found that Ge atoms aggregate on rectangle or square bases whose edges become along $<100>$ directions in the Si substrate. Moreover, it has determined that there is a structure called hut cluster whose facets of $\mathrm{Ge}$ surface might be especially composed of $\{105\}$ planes, though the atoms are crystallographically reconstructed. After further investigations, using STM and quantum calculation, the pyramidal structural model of QD with $\{105\}$ facets has been constructed $[1,8]$.

Therefore, in this article, using molecular dynamics (MD), we estimate the mechanical properties in the structure of self-assembled QD in $\mathrm{Ge} / \mathrm{Si}(001)$ system. We are focusing on the Ge hut clusters which have observed in previous researches [1,7]. There are two main species of hut clusters: pyramids and wedges. We are to computationally model pyramidal one because it has higher symmetry and simpler configuration. We will discuss stress and strain distribution and size dependency of mechanical properties in the pyramidal structure. The knowledge of QD's mechanical properties that is obtained by MD in this study contributes to electron state control and strength design of QDs. This serves as guidelines for developing QD.

This paper is organized as follows. First, we formulate atomic strain measure and atomic stress, and show computational setup and conditions for pyramidal QD structure. Then, the results of MD simulations are shown and discussed. Finally, we show conclusions for the mechanical properties in pyramidal hut clusters of QDs.

\section{Theory}

\subsection{Empirical Interatomic Potential}

In this study, the interatomic potential of Tersoff type (T3) is used, in which the potential is capable to describe the covalent bonding in diamond structure such as those of silicon and germanium crystal. In the formulation of this potential, bond strength between atoms implicitly depends on coordination number of each atom and experimental binding energy obtained as cohesive one is integrated. Tersoff potential is superior to other manybody potentials as for accurate reproduction of the elastic properties [9]. Here, we use potential parameters given by former reference [10].

\subsection{Atomic Strain Measure}

In order to estimate the strain in the three-dimensional structure using computational result obtained by molecular dynamics (i.e. atomic coordinates), we introduce herewith an idea of strain measure defined in the atomic scale [11].

$$
\varepsilon_{i j}=\frac{1}{2\left(r_{i j}(0)\right)^{2}}\left[u_{i j}^{\alpha} r_{i j}^{\beta}(0)+u_{i j}^{\beta} r_{i j}^{\alpha}(0)\right]
$$

where $\alpha$ and $\beta$ are components $(\alpha, \beta=x, y, z)$. In Equation (1), all of the components except for $u_{i j}{ }^{\alpha}$ or $u_{i j}{ }^{\beta}\left(u_{i j}\right.$ is displacement between atoms $i$ and $j$ ) have been calculated from the reference (undeformed) atomic configuration, $r_{i}^{\alpha}(0)$. In order to evaluate strain at individual atomic sites, Equation (1) is simply averaged over the neighboring atoms with which the atom $i$ interacts, as follows:

$$
\varepsilon_{i}^{\alpha \beta}=\frac{1}{N_{\text {neighbor }}} \sum_{j=1}^{N_{\text {neighbor }}} \varepsilon_{i j}^{\alpha \beta}
$$

where $N_{\text {neighbor }}$ is the number of neighbors. The value obtained by Equation (2) shows a local and atomic strain around the atom $i$. We call it atomic strain measure (ASM) of the atom $i$.

The atomic strain of $\mathrm{Si}$ and Ge should be calculated on the basis of each equilibrium lattice strain. However, the initial atomic configuration of simulation model is basically constructed by using lattice constant of Si. So, the reference atomic distance $r_{i j}(0)$ is adjusted properly in calculating the atomic strain of Ge using Equation (2). Since actually lattice constant of $\mathrm{Ge}$ is $4.2 \%$ longer than that of $\mathrm{Si}, r_{i j}(0)$ is modified as follow:

$$
\left.r_{i j}(0)\right|_{\mathrm{Ge}-\mathrm{Ge} \text { or Ge-Si }}=r_{i j}(0) \times 1.042
$$

\subsection{Atomic Stress for Triplet Potential}

Stress of an atom is evaluated by derivative of increase of potential energy with regard to strain. Based on solid mechanics and elasticity, this potential energy is supposed to be identical to the strain energy which has been stored by deformation process. The strain is approximately obtained from directional vector between atoms, $r_{i j}$, by assuming uniform strain field in the deformation. In the MD method, atomic stress is strictly formulated for pairwise interaction (from virial theorem) and is generally calculated as for pairwise potential. However, Tersoff potential includes three-body term. So, it is required that atomic stress for triplet of atoms is approximately defined as an average value of pairwise (virial) quantity inside triplet, which is the product of vectors between each two atoms.

$$
\begin{aligned}
\sigma_{i}^{\alpha \beta}= & \frac{1}{2 \Omega_{i}} . \\
& \sum_{j \neq i, k} \sum_{k \neq i, j}\left(\frac{\partial E_{t o t}}{\partial r_{i j}} \frac{r_{i j}^{\alpha} r_{i j}^{\beta}}{r_{i j}}+\frac{\partial E_{t o t}}{\partial r_{i k}} \frac{r_{i k}^{\alpha} r_{i k}^{\beta}}{r_{i k}}+\frac{\partial E_{t o t}}{\partial r_{j k}} \frac{r_{j k}^{\alpha} r_{j k}^{\beta}}{r_{j k}}\right)
\end{aligned}
$$




$$
r_{j k}=r_{i k}-r_{i j}
$$

where $\Omega_{i}$ is an ideal volume per atom in reference configuration and $E_{\text {tot }}$ is total energy of atomic system.

\section{Computation Model}

In the $\mathrm{Si}-\mathrm{Ge}$ system, the nanostructure with $\{105\}$ crystalline facets is observed in the early stage of epitaxial growth of germanium (Ge) atoms. This characteristic structure is generally called a hut cluster. Hut clusters are put into two major categories, pyramids and wedges [1]. The pyramid one is computationally modeled in this study. Figure 1 shows a schematic drawing of geometrical design for the computation model which is originally and actually confirmed by previous STM observation [7]. The computation model is consists of Si substrate and Ge atomic layers. The latter is called wetting layer (WL) being a few ML thick, where $1 \mathrm{ML}$ corresponds to the thickness of one atomic layer, i.e., one half of lattice constant. The pyramidal-shaped cluster is formed on the WL. Both $\mathrm{Si}$ and Ge crystals have diamond structures though the lattice constant of Ge crystal is set up with that of Si crystal. As a result, initial configuration of Ge crystal possesses compressive residual stress and/or strain. Periodic boundary conditions are applied in $x$ - and $z$-directions so that the system is modeled on infinite two-dimensional superlattice. The $2 \mathrm{ML}$ thickness of $\mathrm{Si}$ substrate on the bottom is supposed to be rigid body and their atomic displacements are to be all frozen. Figure 2 shows the computation model labeled A created as stated above. Table 1 shows computational parameters of MD simulation. To investigate size dependency of mechaniccal property of QD, we also calculate models $\mathrm{B}$ and $\mathrm{C}$ having different sizes from $\mathrm{A}$. In each model, the height of pyramidal structure is $8 \mathrm{ML}, 12 \mathrm{ML}$ and $16 \mathrm{ML}$, respectively. WL is $4 \mathrm{ML}$ thick. These models are used as initial atomic configuration for MD simulation.

In order to analyze the strain in the structure, we first need to obtain a stable crystal structure. The calculation procedures are as follows:

1) Structural relaxation (100.0 ps) from the initial configuration without control of system temperature (equilibration of the structure).

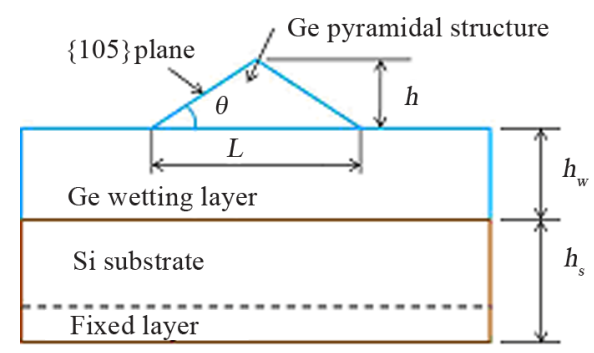

Figure 1. The schematic drawing of computation model (geometrical design).
2) The system temperature is dropped down to $300 \mathrm{~K}$ for relatively long steps, by using conventional velocity scaling method.

3) Equilibrium calculation (20.0 ps) with the control temperature.

Then, finally, we obtain stable crystal structure. Table 2 shows the detail of these relaxation procedures.

During the procedure 3), we calculate atomic strain measure (ASM) and atomic stress of individual atoms by using the formulation, Equation (2) and Equation (4), described in Section 2 above. ASMs are measured with regard to reference configuration of atoms and are calculated from atomic configuration at any time. Since atoms are moving all the time, the ASM and atomic stress of

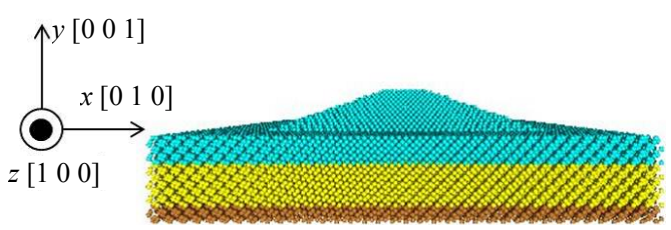

Figure 2. The initial atomic configuration of MD simulation model A.

Table 1. The model parameters used for MD simulation.

\begin{tabular}{cccc}
\hline Model & A & B & C \\
\hline Cell size $x, y$ direction $[\mathrm{nm}]$ & 20.098 & 25.530 & 30.961 \\
Cell size in $y$ direction $[\mathrm{nm}]$ & 6.215 & 25.530 & 30.961 \\
Base length of the pyramid $L[\mathrm{~nm}]$ & 13.580 & 16.295 & 21.727 \\
Height of the pyramid $h[\mathrm{~nm}]$ & 2.173 & 3.259 & 4.345 \\
Height of Ge wetting layer $h_{w}[\mathrm{~nm}]$ & & 1.086 & \\
Height of Si substrate $h_{s}[\mathrm{~nm}]$ & & 2.173 & \\
$\quad$ The number of atoms & 76134 & 132076 & 209009 \\
The number of atoms at the pyramid & 7684 & 21626 & 46559 \\
The number of Ge atoms at the & 21904 & 35344 & 51984 \\
$\quad$ wetting layer & 46546 & 75106 & 110466 \\
The number of Si atoms & & 11.3 & \\
The angle $\theta$ [deg.] & & & \\
\hline
\end{tabular}

Table 2. The model parameters used for relaxation calculation.

\begin{tabular}{ccccc}
\hline Initial temperature $[\mathrm{K}]$ & \multicolumn{3}{c}{300.0} \\
\hline Time increment $[\mathrm{fs}]$ & & \multicolumn{3}{c}{0.5} \\
\hline & Model & \multicolumn{4}{c}{ Calculation procedure } \\
The number of time steps & A & & $(1)$ & $(3)$ \\
& B & 50,000 & 16,140 & \\
& C & & \multicolumn{3}{c}{17,400} & 10,000 \\
Temperature gradient $[\mathrm{K} / \mathrm{s}]$ & & \multicolumn{3}{c}{$-5.0 \times 10^{12}$} \\
\hline
\end{tabular}


individual atom largely fluctuates at every computational step. Therefore, we impose a time average to ASM and atomic stress of individual atom so as to exclude unimportant fluctuation and make clearer evaluation. Here, we focus on the ASM (components $\varepsilon_{x x}$ and $\varepsilon_{y y}$ ) of individual atoms along the center axis of pyramidal structure.

\section{Result and Discussion}

Figures 3 and 4 show the distribution of the ASM, viewing on the cross-section parallel to $x y$ plane. These are for the $\varepsilon_{x x}$ component in the direction of [010] and for the $\varepsilon_{y y}$ components in the direction of [001], respectively. As shown in Figure 3, $\varepsilon_{x x}$ is almost zero in top of the pyramidal structure. It means that strain relaxation occurs in the pyramidal structure. In addition to that, relatively large compressive strain is observed in the bottom edge of the pyramidal structure. Figure 5 shows the schematic of local compressive strain. This local compressive strain observed in edge of the pyramidal structure is understood as a residual strain which is caused by elastic deformation in $x$ direction ([010]) in the pyramidal structure and by strong constraint from Si substrate in WL under the pyramidal structure. As shown in Figure 4, a certain large tensile strain is also observed in the region marked by a circle. This is because a stress component perpendicular to the surface ( $y$ direction, [001]) is vanished out, but strain components in other two directions ( $x$ and $z$, [100]) reside in compressive regime and they cause lateral strain in the $y$ direction.

Figures 6 and 7 show the distribution of ASM for the $\varepsilon_{x x}$ and for the $\varepsilon_{y y}$ on the center line of the pyramid. The value of abscissa corresponds to 0 - $8 \mathrm{ML}$ for Si substrate (0 - $2 \mathrm{ML}$ for fixed atoms), 9 - $12 \mathrm{ML}$ for $\mathrm{WL}$, and 13 ML and larger for pyramidal structure of hut cluster, respectively. As shown in Figure 6, the largest compressive ASM of Ge atom in $9 \mathrm{ML}$ (at the interface betweenWL and Si substrate) is found. This is reasonable because these Ge atoms and Si atoms join coherently and $\mathrm{Ge}$ atoms are subject to constraint from $\mathrm{Si}$ substrate at the bottom. At the top of pyramidal structure, strain marks peak value due to atomic rearrangement in the surface. As shown in Figure 7, pyramidal structure and WL expand in $y$ direction by compressive elastic strain in $x$ and $z$ directions.

Next, we discuss the size dependency of pyramidal structure. Figure 8 shows the relation between $\varepsilon_{x x}$ and the position normalized by the height of WL and pyramidal structure. As shown in Figure 8, the distribution of $\varepsilon_{x x}$ is identical in every model. Other components of ASM are obtained in similar trend as $\varepsilon_{x x}$. Accordingly, ASM distribution in pyramidal structure and WL is three-dimensionally identical and is independent of the size of pyramidal structure. Furthermore, in spite of atomic structure, the ASM is continuous smoothly even at the inter- face between the pyramidal structure and WL.

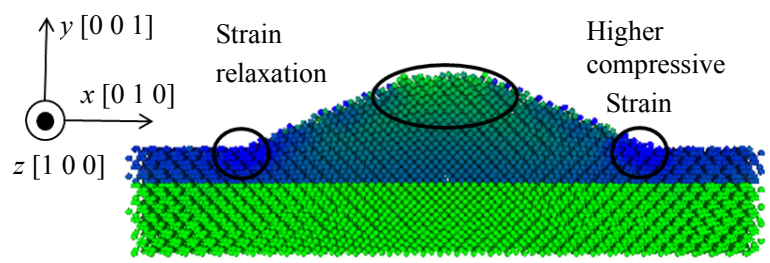

Figure 3. Distribution of ASM component $\varepsilon_{x x}$ (cross-section onto $x y$ plane).

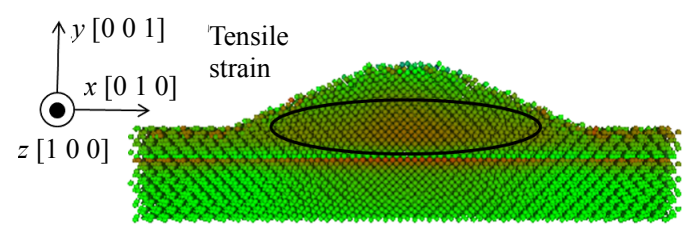

Figure 4. Distribution of ASM component $\varepsilon_{\mathrm{yy}}$ (cross-section onto $x y$ plane).

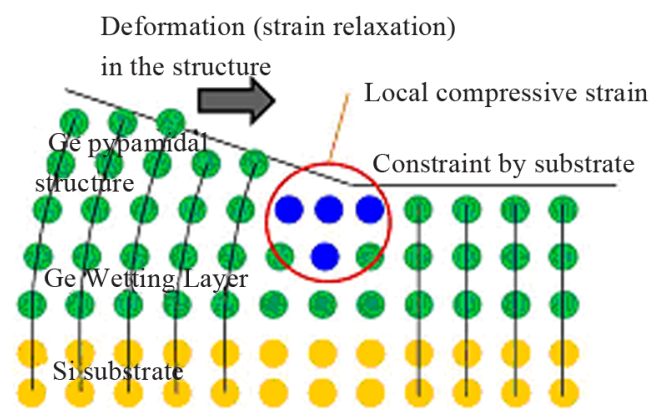

Figure 5. Residual compressive strain in the edge of pyramidal structure.

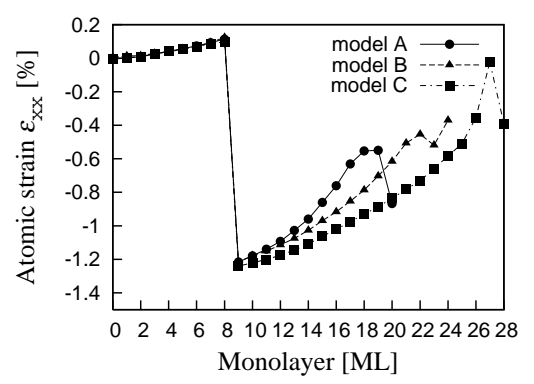

Figure 6. ASM distribution at every 1 ML: $\varepsilon_{x x}$ component.

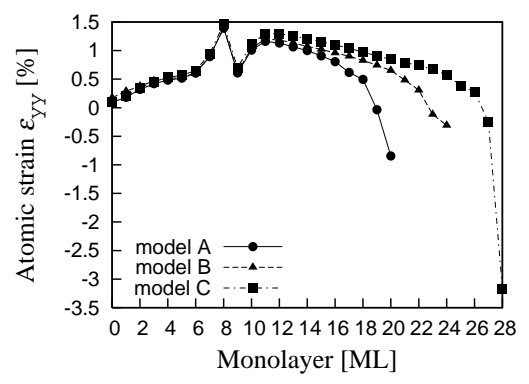

Figure 7. ASM distribution at every 1 ML: $\varepsilon_{\mathrm{yy}}$ component. 


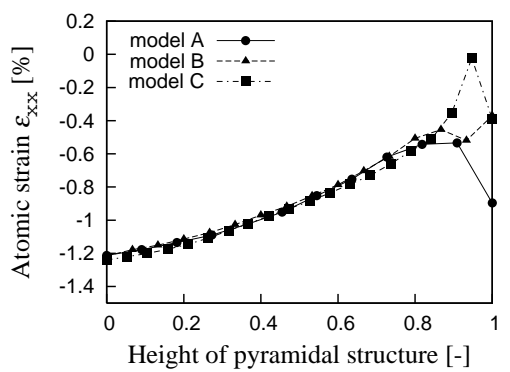

Figure 8. Normalized ASM distribution of each 1 ML in WL and pyramidal structure: $\varepsilon_{x x}$ component.

In order to discuss in detail distribution of stress and strain in pyramidal structure and WL, we also calculate hydrostatic stress and volumetric strain and focus on size dependency and correlation of them. Hydrostatic stress of individual atoms is calculated from atomic stress components,

$$
\sigma_{i}^{m}=\frac{\sigma_{i}^{x x}+\sigma_{i}^{y y}+\sigma_{i}^{z z}}{3}
$$

Figures 9 and 10 show hydrostatic stress $\sigma^{m}$ at the surface of pyramidal structure and the interface between Ge WL and Si substrate (in the following, it is called "Ge-Si interface") beneath the pyramidal structure, respectively.

In these diagrams, hydrostatic stress $\sigma^{m}$ is plotted for atomic coordinate normalized by the length $L$ of pyramiddal structure as abscissa. As shown in Figures 9 and 10, positive $\sigma^{m}$ (i.e. tensile stress) occurs at the surface of pyramidal structure. On the other hand, negative $\sigma^{m}$ (i.e. compressive stress) occurs at Ge atoms in Ge-Si interface. Compressive hydrostatic stress at the Ge-Si interface is largely owing to the compressive stress in $x$ and $z$ directions, which is caused by lattice mismatch between Ge WL and Si substrate. On the other hand, tensile hydrostatic stress at the surface of pyramidal structure is brought about by surface tension. Accordingly, some concentration of hydrostatic stress is also observed as a result of surface atomic reconstruction. It is believed that contribution of compressive stress in $x$ and $z$ directions by lattice mismatch to this tensile hydrostatic stress is smaller because of strain (stress) relaxation in pyramidal structure. The distribution of $\sigma^{m}$ is smooth even at the Ge-Si interface, but that at the surface region shows undulation. Atoms in the Ge-Si interface joins coherently. However, the $\{105\}$ facet of pyramidal structure has atomic-scale surface step. The undulation of $\sigma^{m}$ at the surface of pyramidal structure is caused by the variation of coordination number of each atoms due to this surface-step structure.

As shown in Figures 9 and 10, distribution of hydrostatic stress normalized by length $L$ at the surface and Ge-Si interface is identical, except for the center region.

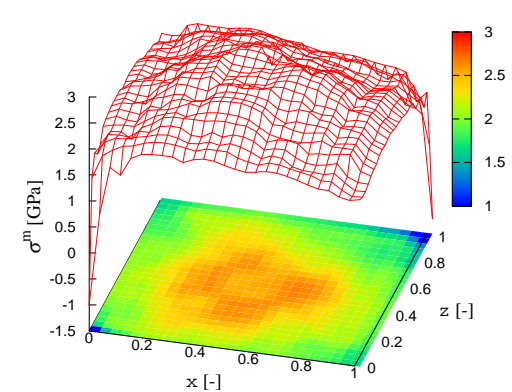

(a) $h=2.173 \mathrm{~nm}$

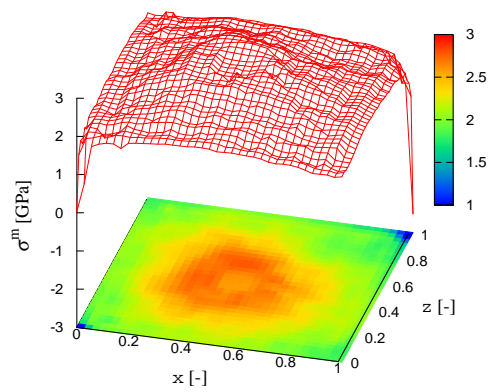

(b) $\mathrm{h}=3.259 \mathrm{~nm}$

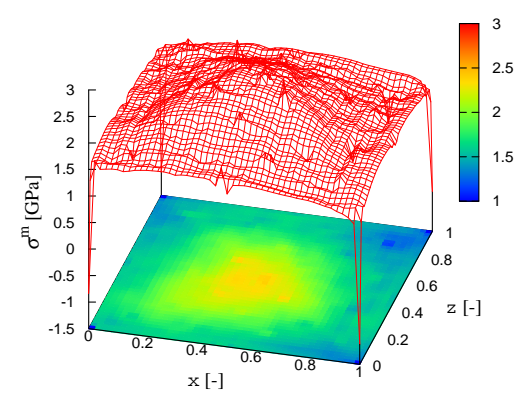

(c) $\mathrm{h}=4.345 \mathrm{~nm}$

Figure 9. The distribution of hydrostatic atomic stress $\sigma^{m}$ at surface of pyramidal structure (atomic coordinate normalized base length $L$ plot along the abscissa).

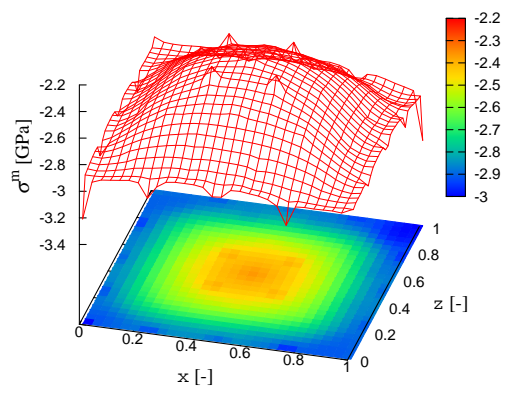

(a) $\mathrm{h}=2.173 \mathrm{~nm}$

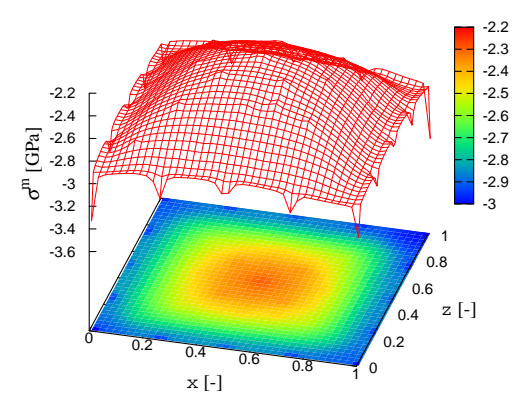

(b) $\mathrm{h}=3.259 \mathrm{~nm}$

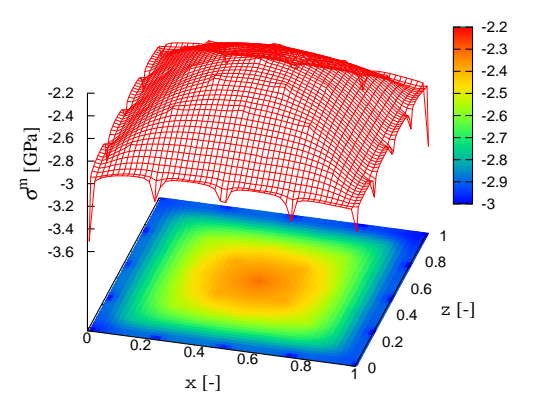

(c) $\mathrm{h}=4.345 \mathrm{~nm}$

Figure 10. The distribution of hydrostatic atomic stress $\sigma^{m}$ at Ge in between Ge WL and Si substrate under the pyramidal structure (atomic coordinate normalized base length $L$ plot along the abscissa). 
The reason why the difference at the center occurs is that the apex of pyramidal structure is the same size but is not scaled in total size. However, it is believed that hydrostatic stress at the surface of pyramidal structure and the Ge-Si interface is constant without dependence on size of pyramidal structure.

Figures 11 and 12 show the relationship between hydrostatic stress $\sigma^{m}$ and atomic volumetric strain $\varepsilon^{V}$. Here, atomic volumetric strain is calculated from components of ASM, Figure 11 shows plots for atoms on the center line of the pyramid, just as Figures 6 and $\mathbf{7}$ above. Several plots of positive $\sigma^{m}(>0)$ are found at the surface region, and their distribution seems at random. Figure 12 is a magnified figure of Figure $\mathbf{1 1}$ for its densely plotted area. The relationship between hydrostatic stress and atomic volumetric strain is basically linear for all models, except for the surface of pyramidal structure and $\mathrm{Ge}-\mathrm{Si}$ interface. This means that there is a reasonable correlation between atomic stress proposed in the present study and atomic strain measure, ASM.

\section{Conclusions}

We perform molecular dynamics simulation for investigating mechanical characteristic in an uncapped pyramidal structure in the $\mathrm{Ge} / \mathrm{Si}(001)$ system with lattice mismatch. We estimate the strain by mean of atomic strain measure (ASM) which is formulated on the Green's defi-

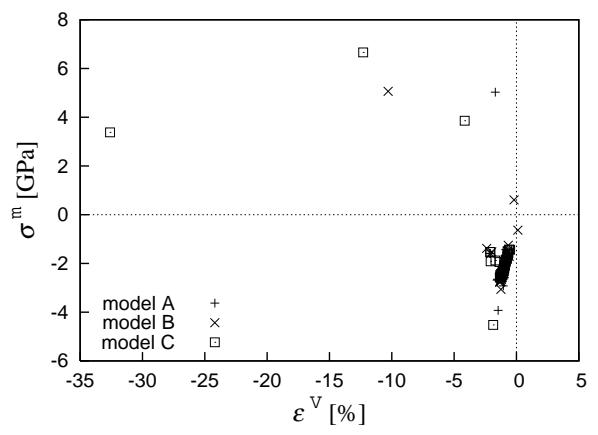

Figure 11. The relationship between hydrostatic stress $\sigma^{m}$ and atomic volumetric strain $\varepsilon^{V}$.

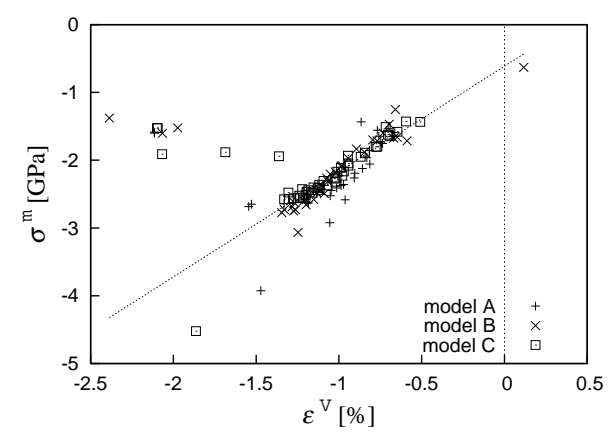

Figure 12. The correlation between hydrostatic stress $\sigma^{m}$ and atomic volumetric strain $\varepsilon^{V}$. nition of continuum strain and is expressed with atomic positions, and atomic stress for triplet interaction. Following results are obtained.

1) Elastic strain and stress in atomic scale can be qualitatively calculated.

2) Local compressive strain in $x$ and $z$ directions is observed in edge of the pyramidal structure. This local compressive strain is understood as a residual strain which is caused by elastic deformation in $x$ and $z$ directions in the pyramidal structure and by strong constraint from Si substrate in WL under the pyramidal structure.

3) Hydrostatic stress at the surface of pyramidal structure and the $\mathrm{Ge}-\mathrm{Si}$ interface is constant without dependence on size of pyramidal structure.

4) There is a reasonable correlation between atomic stress proposed in the present study and atomic strain measure, ASM, except for the surface of pyramidal structure and Ge-Si interface.

\section{Acknowledgements}

This study is partly supported by "Strategic Project to Support the Formation of Research Bases at Private Universities: Matching Fund Subsidy from MEXT (Ministry of Education Culture, Sports, Science and Technology) (2012)".

\section{REFERENCES}

[1] L. V. Arapkina and V. A. Yuryev, "Atomic Structure of Ge Quantum Dots on the Si(001) Surface," JETP Letters, Vol. 91, No. 6, 2010, pp. 281-285. doi:10.1134/S0021364010060056

[2] A. A. Williams, et al., "Strain Relaxation during the Initial Stages of Growth in Ge/Si(001)," Physical Review B, Vol. 43, No. 6, 1991, pp. 5001-5011. doi:10.1103/PhysRevB.43.5001

[3] A. I. Nikiforov, V. A. Cherepanov, O. P. Pchelyakov, A V. Dvurechenskii and A. I. Yakimov, "In situ RHEED Control of Self-Organized Ge Quantum Dots," Thin Solid Films, Vol. 380, No. 1-2, 2000, pp. 158-163. doi:10.1016/S0040-6090(00)01493-0

[4] P. Raiteri, F. Valentinotti and L. Miglio, "Stress, Strain and Elastic Energy at Nanometric Ge Dots on $\mathrm{Si}(001)$," Applied Surface Science, Vol. 188, No. 1-2, 2002, pp. 4-8. doi:10.1016/S0169-4332(01)00702-4

[5] W. Yu and A. Madhukar, "Molecular Dynamics Study of Coherent Island Energetics, Stresses, and Strains in Highly Strained Epitaxy," Physical Review Letters, Vol. 79, No. 5, 1997, pp. 905-908. doi:10.1103/PhysRevLett.79.905

[6] Y. Kikuchi, H. Sugai and K. Shintani, "Strain Profiles in Pyramidal Quantum Dots by Means of Atomistic Simulation," Journal of Applied Physics, Vol. 89, No. 2, 2001, pp. 1191-1196. doi:10.1063/1.1335822

[7] Y.-W. Mo, D.E. Savage, B. S. Swarzentruber and M. G. Lagally, "Kinetic Pathway in Stranski-Krastanov Growth of Ge on Si(001)," Physical Review Letters, Vol. 65, No. 
8, 1990, pp. $1020-1023$. doi:10.1103/PhysRevLett.65.1020

[8] Y. Fujikawa, et al., "Origin of the Stability of Ge(105) on Si: A New Structure Model and Surface Strain Relaxation," Physical Review Letters, Vol. 88, No. 17, 2002, pp. 176101.1-176101.4. doi:10.1103/PhysRevLett.88.176101

[9] H. Balamane, T. Haliciogau and W. A. Tiller, "Comparative Study of Silicon Empirical Interatomic Potentials," Physical Review B, Vol. 46, No. 4, 1992, pp. 2250-2279. doi:10.1103/PhysRevB.46.2250
[10] J. Tersoff, "Modeling Solid-State Chemistry: Interatomic Potentials for Multicomponent Systems," Physical Review $B$, Vol. 39, No. 8, 1988, pp. 5566-5568. doi:10.1103/PhysRevB.39.5566

[11] K. Saitoh and W. K. Liu, "Molecular Dynamics Study of Surface Effect on Martensitic Cubic-to-Tetragonal Transformation in Ni-Al Alloy," Computational Materials Science, Vol. 46, No. 2, 2009, pp. 531-544. doi:10.1016/i.commatsci.2009.04.025 